\title{
PIMS: Nach SARS-CoV-2-Infektion aus dem Nichts aufgetaucht
}

Mit PIMS hat die Corona-Pandemie der Pädiatrie eine neue Krankheit beschert, mit der zunächst niemand rechnet, die dann aber häufig dramatisch verlaufen kann. Da die Erkrankung gut behandelbar ist, ist es wichtig, sie so früh wie möglich zu diagnostizieren.

\begin{abstract}
Auch wenn derzeit über immer schneller steigende SARS-CoV-2-Infektionszahlen bei Kindern und Jugendlichen berichtet wird, müssen in Deutschland Patienten dieser Altersgruppe nur selten wegen COVID-19 stationär versorgt werden. Wie Prof. Reinhard Berner, Dresden, beim Pädiatrie-Update 2021 in Berlin berichtete, wurden in der gesamten Pandemiezeit in Deutschland bislang etwa 1000 Kinder in Kliniken behandelt, 50 von innen auf Intensivstationen. Anders als bei den Erwachsenen zeichnet sich bislang noch kein Wiederanstieg der Fallzahlen von COVID-19 auf den Kinderintensivstationen $a b$.
\end{abstract}

\section{Neues Krankheitsbild in der Pädiatrie}

Neben den schweren COVID-19-Fällen wird in den Kliniken allerdings bereits seit April 2020 weltweit ein völlig neues Krankheitsbild beobachtet, das dem Kawasaki-Syndrom ähnelt und als "Pädiatrisches hyperinflammatorisches Syndrom mit Multiorganbeteiligung" (Pediatric Inflammatory Multisystem Syndrome, PIMS) oder Multiinflammatory Syndrome with COVID-19 (MIS-C) bezeichnet wird. Kinder, die diese Krankheit schwer trifft, sind, anders als bei COVID-19 in der Regel nicht durch Vorerkrankungen belastet. Doch das Krankheitsbild von PIMS ist schwerer als das von COVID-19 selbst. Berner berichtete, dass $7 \%$ der Kinder nachweisbare, wahrscheinlich nicht korrigierbare Folgeschäden hätten, die überwiegend das kardiovaskuläre System beträfen. Über die Pathophysiologie von PIMS wird noch weitge- hend spekuliert. Eine Hypothese ist Berner zufolge, dass der HLA-Genotyp eine Rolle im Zusammenhang mit dem Zytokinsturm bei PIMS spielen könnte.

\section{Fallbericht}

Ein Pandemie-Fallbericht: Ein Neunjähriger wird mit septischem Schock auf die Intensivstation aufgenommen. Hier bestätigt sich die zusätzliche Verdachtsdiagnose eines "pediatric inflammatory multisystem syndrome" (PIMS). Im Verlauf zeigt sich deutlich, dass es beim PIMS - auch verzögert zu einer mitunter bedrohlichen Verschlechterung der kardiorespiratorischen Situation kommen kann.

Die Deutsche Gesellschaft für Pädiatrische Infektiologie (DGPI) hatte bis zum 31.03.2021 insgesamt 245 PIMS-Fälle erfasst. Wie Dr. Florian
Hoffmann, München, beim Pädiatrie-Update 2021 berichtete, tritt PIMS mit einer zeitlichen Verzögerung von zwei bis vier Wochen zur SARS-CoV-2-Infektion auf. Viele der an PIMS erkrankten Kinder hatten zuvor nur leichte COVID-19-Symptome, die meisten waren asymptomatisch mit SARS-CoV-2 infiziert, sodass erst der Antikörpernachweis Klarheit brachte.

\section{PIMS: klinische Symptomatik}

Das Hauptsymptom bei PIMS ist hohes Fieber über $40^{\circ} \mathrm{C}$, das sich anders als bei COVID-19 nur schwer senken lässt. Ein Großteil der Kinder leidet an Durchfall und Erbrechen. Im Labor sind Entzündungsmarker wie CRP und Procalcitonin massiv erhöht, häufig besteht gleichzeitig eine relative Lymphopenie. Zum therapierefraktären Fieber kommt
Quelle: basierend auf: Berner, R. Dresden; Hoffmann, F., München. Vorträge beim Pädiatrie-Update, Berlin, 17. April 2021 www

springermedizin.de 21.04.2021 | Pädiatrie Update | Nachrichten

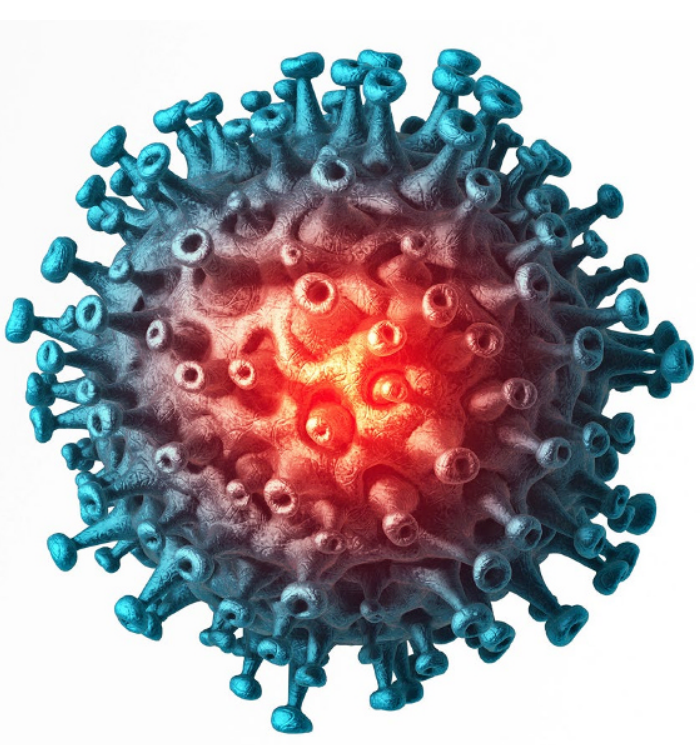

A SARS-CoV-2 Virus: Seit April 2020 wird in der Pädiatrie ein völlig neues Krankheitsbild beobachtet 
bei $76 \%$ der Kinder eine Myokarditis mit Funktionseinschränkung hinzu, $57 \%$ erleiden einen vasoplegischen Schock, bei $56 \%$ besteht ein Exanthem und bei $52 \%$ eine Konjunktivitis. Problematisch sind Hoffmann zufolge vor allem Koronardilatationen und -aneurysmen, die bei einem Viertel der Patienten auftreten. Insgesamt müssen etwa $70 \%$ dieser Kinder mit PIMS auf einer Intensivstation behandelt werden.

》) Bei hohem Fieber und gastrointestinalen Symptomen an PIMS denken!

Während das klassische KawasakiSyndrom in einem Alter von sechs Monaten bis fünf Jahren auftritt, sind PIMS-Patienten meist zwischen vier und 17 Jahre alt und erkranken weitaus schwerer. Das höchste Risiko für einen schweren Verlauf haben Kinder zwischen sechs und zwölf Jahren. Die Abgrenzung zum Kawasaki-Syndrom ist nicht immer einfach, doch Lymphopenie und ein vasoplegischer Schock sowie Exantheme treten bei PIMS weitaus häufiger auf. Auch geht das Kawasaki-Syndrom nur sehr selten mit gastrointestinalen Symptomen einher.

\section{PIMS: Früherkennung ist wichtig!}

Wegen der Gefahr schwerer Organdysfunktionen ist das frühe Erkennen des PIMS wichtig. Alle Patienten, bei denen der Verdacht auf diese Erkrankung besteht, sollten Hoffmann zufolge mit Kortikosteroiden und hochdosierten intravenösen Immunglobulinen behandelt werden. „Die meisten Kinder sprechen auf diese Behandlung fantastisch an", so Hoffmann, und sind nach ein paar Tagen wieder gesund". Besteht eine kardiale oder hämodynamische Manifestation, soll eine hoch dosierte ASS-Therapie ergänzt werden. Bei therapierefraktärem Verlauf und persistierender Inflammation kann eine IL1- oder IL5-Blockade erwogen werden.

"Wir wissen", so Hoffman, „dass die Infektionszahlen mit der Mutante B.1.1.7. auch in Schulen und Kitas in die Höhe schnellen werden. Damit ist auch ein Anstieg der Anzahl von PIMS-Patienten zu erwarten. Seien Sie deshalb bei verdächtigen Symptomen wie Fieberexanthem und gastrointestinalen Beschwerden aufmerksam!"

\section{Autorin:Dr. Christine Starostzik}

Hinweis des Verlags. Der Verlag bleibt in Hinblick auf geografische Zuordnungen und Gebietsbezeichnungen in veröffentlichten Karten und Institutsadressen neutral.

Paediatr. Paedolog

2021·56:134-135

https://doi.org/10.1007/ s00608-021-00901-w (c) Springer-Verlag GmbH Austria, ein Teil von Springer Nature 2021
Hier steht eine Anzeige. Springer 